\title{
CEPHALOMETRIC ANALYSIS OF ADAPTATIONS AFTER LENGTHENING OF THE MASSETER MUSCLE IN ADULT RHESUS MONKEYS, MACACA MULATTA
}

\author{
D. S. Carlson and E. D. Schneiderman \\ Department of Anatomy and Cell Biology and Center for Human Growth and Development, \\ The University of Michigan School of Medicine, Ann Arhor, MI 48109, U.S.A.
}

\begin{abstract}
Summary--This relationship between the function of the muscles of mastication and craniofacial form was investigated in young adult monkeys by increasing the functional length of the elevator muscles of the mandible non-invasively by a bite-opening splint cemented to the maxillary dentition. The major adaptations to increased vertical dimension were (1) marked superior and some anterior displacement of the maxillary complex, (2) dental intrusion and (3) rotation of the mandible. These alterations produced a shortening of the lengthened masseter muscle, i.e. a reduction in the amount of muscle stretch brought about by the appliance. Migration of the masseteric insertion along the ramus did not contribute significantly to the pattern of adaptation. The role of the masseter muscle in craniofacial adaptations to altered vertical dimension was determined by detaching and re-attaching the insertion of the masseter muscle in one group of experimental animals. The myotomized monkeys experienced significantly less anterior displacement of the maxilla than the non-myotomized monkeys, indicating that the surgery may have lessened some of the anteriorly-directed distracting forces of the lengthened masseter. Masseter myotomy alone was not sufficient to eliminate the verticallydirected distracting forces of the remainder of the mandibular elevator muscles brought about by increasing the vertical dimension of the lower face.
\end{abstract}

\section{HNTRODUCTION}

It is well known that the growth of the craniofacial skeleton can be modified experimentally by alteration of the function of the muscles of mastication (Washburn, 1947; Hoyte. 1966; Moore and Lavelle, 1974; McNamara, 1975). The influence of altered muscle function on the fo:m of the craniofacial skeleton in adults, however, is less well understood. This is particularly apparent in considerations of the stability of results after surgical treatment of certain human jaw discrepancies, where correction of anteroposterior and vertical jaw malalignments often involves an increase in the length and an alteration of the position of the muscles of mastication [see Carlson et al. (1982) for an extensive review]. Consequently, many investigators suggest that skeletal relapse after orthognathic surgery, i.e. partial or complete return of the altered skeletal segment after treatment, is primarily the result of adverse muscle tension acting on the repositioned skeletal segment (Ware and Taylor, 1968; Poulton and Ware, 1971; Steinhauser, 1973; Ive et al., 1977; Fpker et al., 1978). Unfortunately there has been no comprehensive, quantitative experimental analysis of the influence of muscle stretch with and without surgery on craniofacial form and muscle position in adults. McNamara (1974) provided a brief report of adaptations in the denio-alveolar region and mandibular joint of adolescent and adult monkeys. Yellich, McNamara and Ungerleider (1981) studied the position of the masseter muscle after lengthening it in adult monkeys. However, the sample sizes in the long-term (24-48 week) treatment groups were small, the system for analysing muscular adaptations was inadequate and dento-alveolar and skeletal adaptations were not considered quantitatively.

Our purpose was to provide a longitudinal, quantitative analysis of the specific morphological adaptations that take place in the craniofacial complex of adult thesus monkeys after increasing the vertical dimension of the lower face with and without masseter myotomy. Having regard to studies of McNamara (1974) and Ycllich et al. (1981), we put forward three hypotheses: (1) that stretching of the mandibular elevator muscles results in an anterior and superior displacement of the maxillary complex; (2) that displacement of the maxillary complex continues until the masseter muscles have re-established their original length via migration up the ramus; (3) that surgical detachment and re-attachment of the masseter muscle enhances muscle migration and, therefore, mollifies or eliminates any tendencies for maxillary displacement.

\section{MATERIALS AND METHODS}

\section{Experimental animals}

Twenty-five adult female rhesus monkeys (Macaca mulatta) were divided into two experimental groups and a control group. Based on the presence of a complete permanent dentition (Hurme and Van Wagenen, 1961) and degrees of occlusal wear (Gantt, 1979), the ages ranged from approx. 6 to $9 \mathrm{yr}$.

\section{Experimental design}

Implant techniques. Radio-opaque tantalum implants were placed in specified regions of the frontal 
bone, zygomaxillary process, maxilla, mandible and cranial base at least 10 weeks prior to the start of the experiment to facilitate cephalometric analysis of bone remodelling and displacement (Plate Fig. 1A). Radio-opaque gold markers composed of root canal broach $2.5-3.0 \mathrm{~mm}$ in length and $0.7 \mathrm{~mm}$ in diameter were inserted percutaneously into the distal region of the masseter muscle at the time of bone marking in order to permit alterations in muscle length and position to be determined using radiographic methods. McNamara et al. (1978) showed that no more than 1.0 $\mathrm{mm}$ variation in the position of the muscle markers occurs after a 6-8 week period of stabilization. The experimental period for each animal was not begun until the muscle markers were judged to be stable as indicated by superimposition of serial radiographs.

Appliance design. At the start, each experimental animal had an intraoral occlusal appliance, designed to create a $15-18 \mathrm{~mm}$ opening interincisally, cemented to its maxillary dental arch (Fig. 1B). This amount of mandibular depression resulted in approx. a 10-15 per cent increase in the distance between the origin and insertion, i.e. stretch, of the masseter muscle, but did not much diminish oral function; animals had no trouble in eating a normal diet of commercial monkeyfood and fruit and in exhibiting normal agonistic display behaviour with the bite-opening appliance in place.

Experimental groups. Two experimental groups were defined, Group A $(n=10)$ and Group ADR $(n=9)$. Group A animals received the bite-opening appliance only. Group ADR animals received the bite-opening appliance and then underwent surgery under aseptic conditions to detach and reattach the masseter muscles bilaterally.

Surgical procedure. With the bite-opening appliance in place, animals in Group ADR were sedated using phencyclidine $\mathrm{HCl}$ and then pentobarbital sodium IV was administered to achieve a surgical level of anaesthesia. A small incision was made along the margin of the angle of the mandible, the platysma muscle was blunt dissected and the masseter muscle exposed. The masseter muscle was sharp dissected from the area of the mandibular angle and was then detached completely from the ramus using a periosteal elevator. After haemostasis was achieved, the masseter muscle was re-approximated along the ramus and sutured to the remaining connective tissue of the pterygomasseteric sling at its stretched position using 4.0 gauge polyglycolic acid suture. The platysma muscle and skin were then closed separately. The same procedure was repeated on the other side.

Cephalometric analysis. Lateral radiographic cephalograms were taken of each animal with the teeth in centric occlusion or, during the experimental period, with the mandibular dentition occluded with the bite-opening appliance. Group A animals were radiographed immediately before (interval A) and immediately after cementation of the appliance (interval 0 ) and 12, 24 and 48 weeks thereafter. Animals in Group ADR were radiographed immediately before appliance placement (interval $\mathrm{A}$ ), immediately before surgery with the appliance in place (interval B) and immediately after surgery (interval 0 ). Each animal was then radiographed 12,24 and 48 weeks after the start of the experiment.
Cephalograms were traced and digitized to record the coordinates of the radio-opaque bone and muscle markers as well as of 58 anatomic landmarks and reference points (Carlson et al., 1982). These data were entered into the University of Michigan's computer system for graphic and quantitative analysis. Two planes of reference, one for the cranium (CRL) and one for the mandible (MRL), were constructed for superimposition of serial cephalographs. These reference lines and perpendiculars through specified bone markers define two coordinate systems originating in the stable cranial base of mandibular bone implants themselves. The systems were manipulated geometrically with the computer so as to remain parallel to the original maxillary and mandibular occlusal planes throughout the experiment. Skeletal adaptations were evaluated by measuring changes between serial cephalograms in the horizontal and vertical positions of the frontal, maxillary, premaxillary and zygomaxillary bone markers relative to the cranial reference line.

Masseter muscle length was defined as the distance between the zygomaxillary implant, indicating the most anterior attachment of the masseter muscle at its origin and the most distal marker in the masseter muscle. The horizontal and vertical position of the masseter muscle markers relative to the mandibular reference line and the cranial reference line was used to describe the location of the masseter muscle at each experimental interval. Dento-alveolar adaptations within the mandible were assessed by measuring the perpendicular distance between the MRL and the posterior and anterior corners of the occlusal splint with the jaws closed.

Statistical analysis. Serial changes in each of the cephalometric variables were calculated for each anjmal at each interval. One-way analysis of variance was used to evaluate the significance of any differences between groups at each experimental interval separately. Paired $t$-tests were used to determine whether any changes within each experimental group between serial intervals were significant. A least squares regression analysis was used to determine whether adaptations within the maxillary complex after increasing the height of the lower face might vary as a function of age.

\section{RESULTS}

\section{Skeletal adaptations (Table 1)}

Frontal bone. The mean change in spatial position of the frontal bone markers between experimental intervals in all groups was less than $0.2 \mathrm{~mm}$ horizontally and vertically. None of the differences in frontal bone position among the groups were statistically significant. This stability of the frontal bone markers indicated that the measuring technique was adequate and that the cranial base was not growing at the time of the experiment.

Maxillary complex. The maxillary complex in control animals remained stable, although there was a small amount of inferior and anterior movement which can be attributed to normal growth in young adults (Schneiderman and Carlson, 1981). The maxillary complex in all experimental animals, on the other hand, underwent a marked displacement in a superior and anterior direction. This displacement was most 

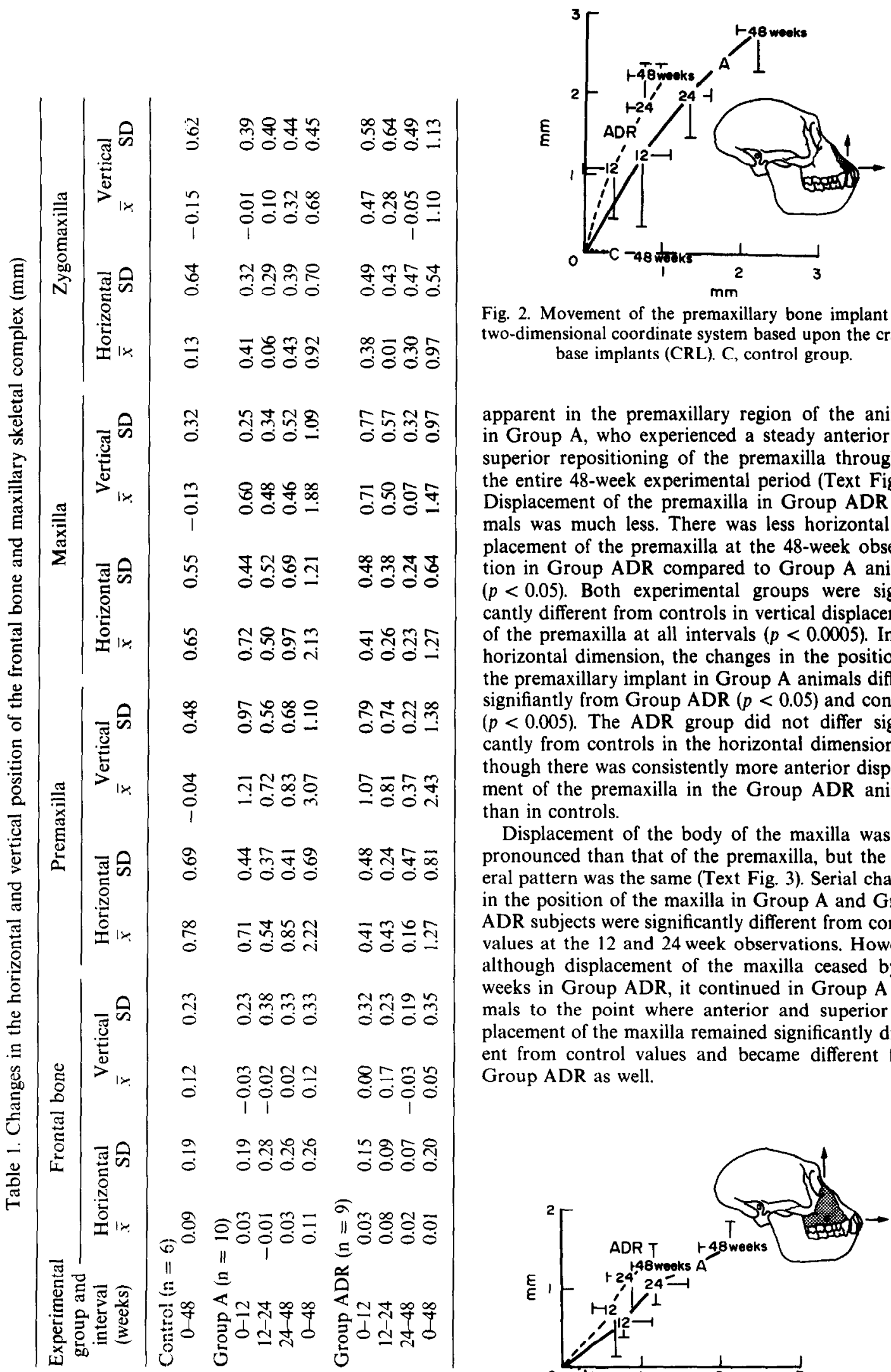

Fig. 2. Movement of the premaxillary bone implant in a two-dimensional coordinate system based upon the cranial base implants (CRL). C, control group.

apparent in the premaxillary region of the animals in Group A, who experienced a steady anterior and superior repositioning of the premaxilla throughout the entire 48-week experimental period (Text Fig. 2). Displacement of the premaxilla in Group ADR animals was much less. There was less horizontal displacement of the premaxilla at the 48-week observation in Group ADR compared to Group A animals $(p<0.05)$. Both experimental groups were significantly different from controls in vertical displacement of the premaxilla at all intervals $(p<0.0005)$. In the horizontal dimension, the changes in the position of the premaxillary implant in Group A animals differed signifiantly from Group ADR $(p<0.05)$ and controls $(p<0.005)$. The ADR group did not differ significantly from controls in the horizontal dimension, although there was consistently more anterior displacement of the premaxilla in the Group ADR animals than in controls.

Displacement of the body of the maxilla was less pronounced than that of the premaxilla, but the general pattern was the same (Text Fig. 3). Serial changes in the position of the maxilla in Group A and Group ADR subjects were significantly different from control values at the 12 and 24 week observations. However, although displacement of the maxilla ceased by 24 weeks in Group ADR, it continued in Group A animals to the point where anterior and superior displacement of the maxilla remained significantly different from control values and became different from Group ADR as well.

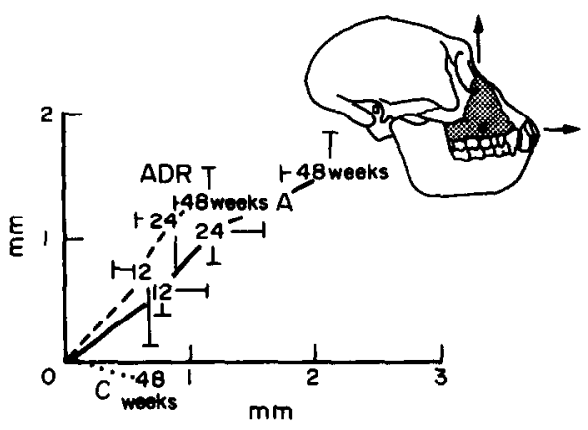

Fig. 3. Movement of the maxillary bone implant relative to the CRL. 


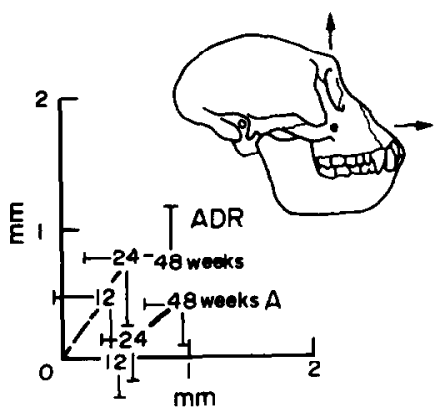

Fig. 4. Movement of the zygomaxillary bone implant relative to the CRL. Changes in the control group are extremely small and therefore are not illustrated.

Zygomaxillary process. The zygomaxillary implant showed the least overall displacement of the implants in the maxillary complex throughout the 48-week experimental period (Text Fig. 4). Over the first 24 weeks of the experiment, in Group A the zygomaxillary process was remarkably stable. However, over the last 24 weeks of the experiment in Group A, the zygomaxillary implant was displaced superiorly and anteriorly $(p<0.05)$. Group ADR animals, on the other hand, underwent a marked superior displacement of the zygomaxillary process during the first 24 weeks of treatment, then became stable with respect to vertical changes but continued to be displaced anteriorly through the 48 -weeks $(p<0.05)$.

\section{Muscle adaptations (Table 2)}

Masseter length. Cementation of the bite-opening appliance resulted in an immediate increase in masseter length of approx. $5.0 \mathrm{~mm}$, i.e. the masseter was stretched by approx. 10-15 per cent beyond its original length (Text Fig. 5). Surgical detachment and reattachment of the masseter in Group ADR immediately halved the amount of stretch brought about by the appliance, so that the masseter muscle at the start of treatment in Group ADR animals was approx. $2.0 \mathrm{~mm}$ longer than it was prior to cementation of the appliance.

During the first 24 weeks, in animals in Group A there was a steady decrease in the length of the masseter muscle until it had shortened on average by $1.5 \mathrm{~mm}$, or approx. 30 per cent of the $5.4 \mathrm{~mm}$ increase in length brought about by the appliance. This change in masseter length between the start of the experiment and the 24-week period was significant $(p<0.01)$. The masseter muscle remained the same length over the last 24 weeks in Group A. In Group ADR, the mean length of the masseter muscle appeared to increase slightly during the first 12 weeks and then to decrease on average by just over $0.5 \mathrm{~mm}$ by 48 weeks. However, none of the the differences in muscle length throughout the experimental period were significant at the 5 per cent level in Group ADR.

Masseter position. A complex picture emerges when changes in the position of the masseter muscle are considered. Relative to the cranium (Text Fig. 6A), the bite-opening appliance resulted in a posterior and slightly inferior displacement of the muscle insertion in both experimental groups. Detachment and reattachment of the masseter in Group ADR resulted in an immediate displacement of the muscle superiorly and anteriorly so that the total amount of stretch was less. During the 48-week period, there was a change in the position of the muscle markers such that the masseter muscle appeared to migrate anterosuperiorly toward its original location on average by $3.0 \mathrm{~mm}$ for Group A and $2.0 \mathrm{~mm}$ for Group ADR.

The only valid way to assess whether migration of the masseter muscle on the ramus took place, however, is to examine the position of the muscle implant relative to the mandibular bone implants using the mandibular reference line. In the control monkeys, there was a small but significant posterior migration of the masseter muscle relative to the MRL over the 48 week period $(p<0.01)$. Analysis of the movement of the muscle markers relative to the MRL in Group A revealed that the masseter muscle underwent a transient proximal displacement that was probably due to the initial muscle stretch, and then underwent slight anterior movement (Text Fig. 6B). There was no significant vertical movement of the muscle marker. The masseter muscle in Group ADR became repositioned superiorly and slightly posteriorly relative to the MRL as a result of the appliance and surgery. It then moved inferiorly during the first 12 weeks, probably shortly after surgery during the process of reattachment to the ramus, where it remained stable. The mean position of the insertion did not change significantly between the 12-24 and 24-48 week intervals in Group ADR. Thus, with the exception of a small but significant amount of anterior movement of the masseter muscle in Group A during the 48 weeks and the displacement of the masseter muscle as a result of the detachment and re-attachment in Group ADR, there was no significant evidence for migration of the masseter muscle in either group throughout the experiment.

\section{Dental intrusion}

By examining the vertical position of the anterior and posterior corners of the occlusal surface of the bite-opening appliance relative to the MRL, it was posssible to estimate the extent to which the buccal segment of the mandibular dentition was intruded. In Group $A$, the premolars were intruded a mean distance of approx. $1.7 \mathrm{~mm}(\mathrm{SD} \pm 0.6)$ and the molars by approx. $3.3 \mathrm{~mm}(\mathrm{SD} \pm 1.0$ ). The premolars and molars were intruded an average of $1.6 \mathrm{~mm}(\mathrm{SD} \pm$ 1.0) and $2.3 \mathrm{~mm}$ ( $S D \pm 1.3$ ), respectively, in Group ADR. These amounts of dental intrusion were significantly different from the small eruptive movements (approx. $0.6 \mathrm{~mm}$ ) in the control animals $(p<0.05$ ). The amount of intrusion of the premolars was not significantly different between Group A and Group ADR. However, the difference in the molar region was closer to significance $(p=0.12)$.

\section{Effects of age}

Least-squares regression analysis demonstrated a significant relationship between estimated age based on dental wear and amount of anterosuperior distraction of the maxillary complex in Group A $(r=0.74$, $p<0.05$ ); the older the monkey the lesser the extent of maxillary displacement. No significant relationship between age and maxillary distraction was found among Group ADR animals. 


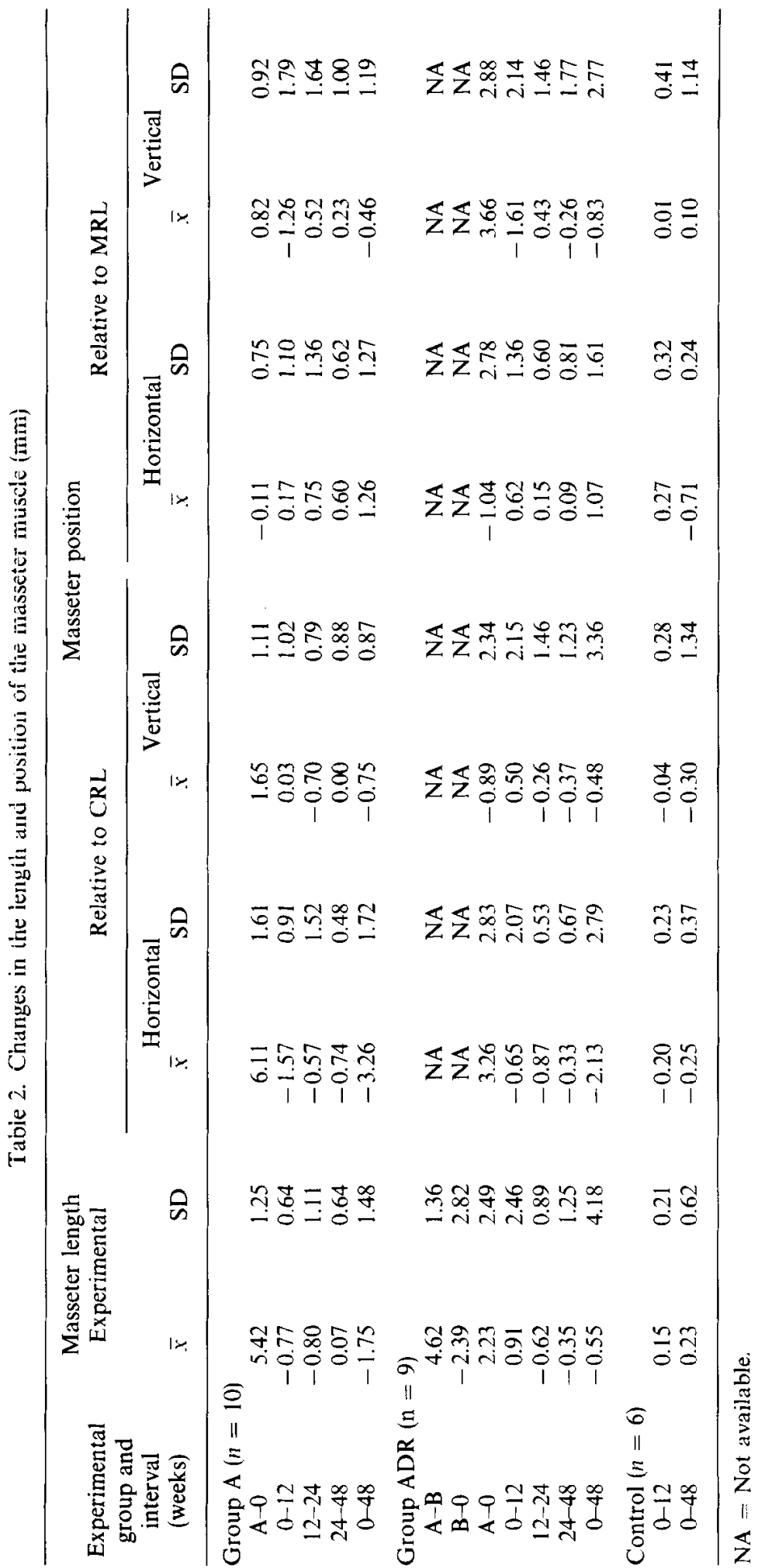




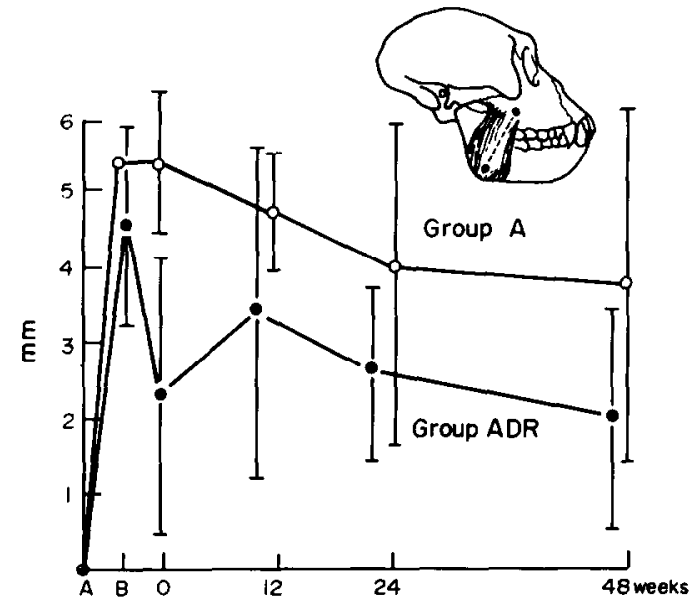

Fig. 5. Changes in the length of the masseter muscle after cementation of the appliance as measured radiographically from the zygomaxillary bone marker to the masseter muscle marker. (A), Pre-appliance; (B), immediately postappliance cementation (Group A) and post-appliance pre-surgery (Group ADR); (0), immediately post-appliance cementation (Group A) and immediately post-surgery (Group ADR). Cumulative mean changes and corresponding 95 per cent confidence intervals are indicated. Change in the negative direction signifies shortening.

\section{DISCUSSION}

The findings indicate that a hierarchy of related adaptations take place within the craniofacial complex after increasing the height of the lower face with and without surgery. Increasing the length of the elevator muscles of the mandible by stretching them $10-15$ per cent beyond their normal resting length without surgery resulted in a steady anterior and superior translation of the premaxilla and maxilla throughout the entire experimental period as well as an intrusion of the mandibular dentition in the molar region. On the other hand, the region of the zygomaxillary process, where the masseter muscle originates, underwent only slight anterior displacement and no superior displacement during the first 24 weeks in Group A. At the same time, during the first 24 weeks, there was a decrease in the stretched length of the masseter muscle. Although consideration of the position of the masseter muscle relative to the cranium suggested that this was due to migration of the masseter musle in an anterior and superior direction after being lengthened, superimposition on the mandibular implants showed that little change in muscle position took place. These results indicate that the decrease in the stretched length of the masseter in Group A during the first 24 weeks of the experiment was due principally to two related factors: (1) anterior and superior translation of the premaxilla and maxilla while the zygomaxillary region remained nearly stationary and (2) intrusion of the buccal segment of the mandibular dentition, both of which allowed the mandible to rotate superiorly relative to the cranial base. Detachment and re-attachment of the masseter muscles in Group ADR led to a slightly different pattern of adaptation, in which there was marked superior translation and little anterior displacement of the entire maxillary complex. No masseter-muscle shortening due to migration took place except as a direct result of surgery.

Two related mechanisms could account for the skeletal and dental adaptations observed. First, increasing or stretching the functional length of the elevator muscles of the mandible by increasing the vertical dimension of the lower face probably resulted, at least initially, in an increase in the habitual tension generated by these muscles both as a result of active muscular contraction and of passive connective tissue forces. Active muscle contraction would result from the muscle-spindle-mediated stretch reflex as the mandibular elevator muscles were lengthened (Botterman, Binder and Stuart, 1978; Houk, 1979; Cooker, Larson and Luschei, 1980). It is known that connective tissues within muscles exert tension when stretched (Yemm and Berry, 1969; Brill and Tryde, 1974; Carlson and Wilkie, 1974). The ability of stretched soft tissues to generate considerable force has been demonstrated
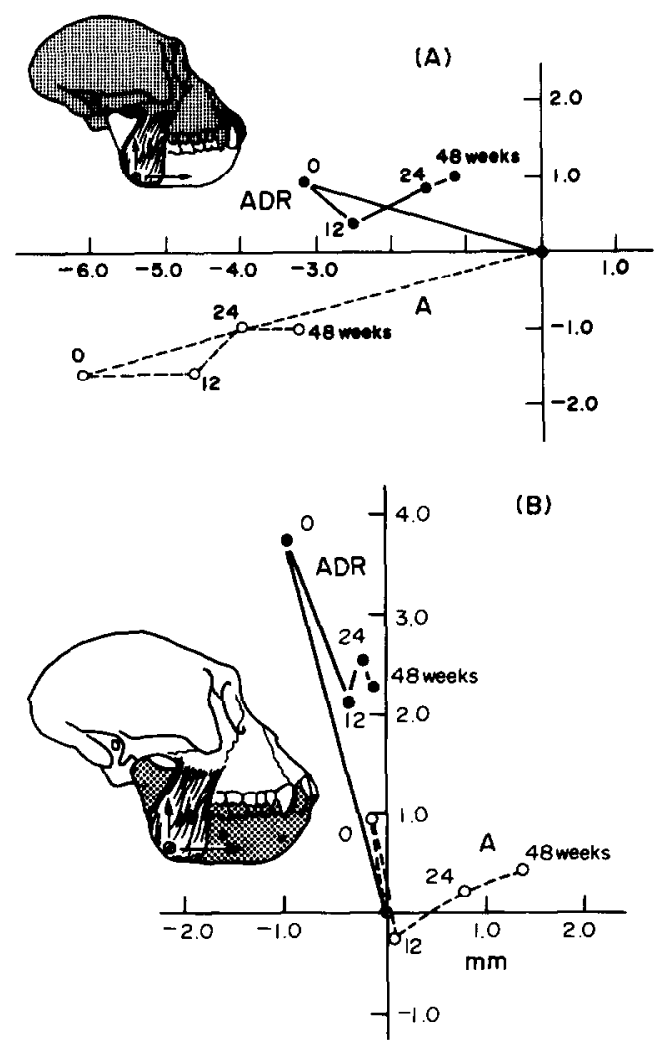

Fig. 6. Changes in the position of the masseter muscle marker relative to the cranial base (A) and mandibular corpus (B) bone markers. Mean change in the position of the muscle marker is charted from the pre-appliance stage (indicated at the origin of the coordinate systems) until 48 weeks. Note that when the changes in masseter muscle position are assessed relative to a coordinate system based upon the cranial implants, there is an appearance of considerable migration towards the original position of the masseter. Alternately, when changes in masseter position are assessed relative to a coordinate system based upon the mandibular implants, the masseteric insertion is seen to move little in any group except as a result of the surgical insert in Group ADR. 


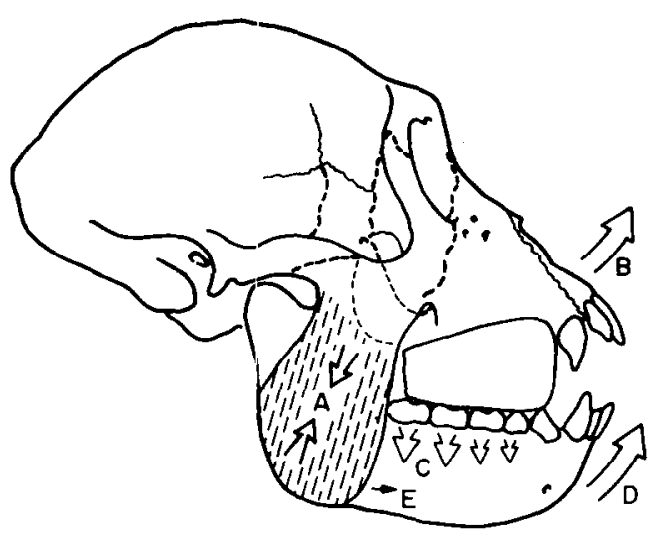

Fig. 7. Summary of adaptations to increased vertical dimension. Stretching of the mandibular elevator muscles brought about by the occlusal splint may increase the tension generated by the muscles (A) and stimulate interstitial, longitudinal growth of the muscle fibres and associated connective tissue. This results in an imbalance of the forces acting on the maxillary skeletal complex, causing it to be displaced anterosuperiorly (B). Adaptive remodelling at the situres of the maxilla and adjacent bones (broken lines) permits this displacement. Increased compressive forces exerted upon the occlusal surfaces of the mandibular post-ranine dention causes intrusion of these teeth $(\mathrm{C})$. These adaptations permit rotation of the mandible (D) resulting in a 30 per cent decrease in the amount of stretch exhibited by the masseter muscle in Group A. At the same time, longitudinal growth of the muscle fibres and of the connective tissue within the muscle probably occurs, resulting in a permanently lengthened muscle and a reduced amount of muscle stretch. The small amount of anterior migration of the insertion of the masseter (E) does not contribute to this shortening.

for the masticatory system of rats by Yemm and Nordstrom (1974). Dechow and Carlson (1982a, b) showed that the depressed mandible exerts superiorly directed forces even when the macaque monkey is completely sedated. Secondly, opening the bite probably resulted in a biomechanical imbalance between muscle function and skeletal form. It is reasonable to assume that the form, size and location of the maxillary skeletal complex is normally maintained within a balanced, homeostatic relationship with the muscles of the craniofacial region. Thus, normal function of the masticatory muscles in the adult would not cause any appreciable change in the form of the maxillary skeletal complex. Ir.troduction of the occlusal splint, however, caused an imbalance in this relationship such that the muscles were re-orientated and probably changed with respect to the forces they produced on a chronic basis, at least until the muscles became adapted to the amount of stretch introduced by the appliance. Thus, we can hypothesize that stretching and re-orientating the mandibular elevator muscles through the use of the occlusal splint resulted in both a transient increase and a directional change in the actively and passively generated forces exerted by these muscles and associated soft tissues that were dissipated through the dentition, occlusal splint and maxillary skeleton. The net result of this increase and re-orientation of force was a displacement of the premaxilla and maxilla in an anterior and superior direction, and intrusion of the buccal segments of the mandibular dentition (Text Fig. 7). The zygomaxillary region underwent little or no superior displacement during the first 24 weeks of the experiment in Group A because it is associated with the origin of the masseter muscle. As no differences in the distance between bone markers in the maxilla and premaxilla were observed during the experimental period, displacement of the maxillary complex must have taken place as a unit, probably as a result of adaptive changes at the zygomaxillary and pterygopalatine sutures. Due to the significant relationship between the amount of maxillary response and age in Group A, it also appears that the ability of these sutures to respond is a function of both treatment and age.

The pattern of craniofacial adaptation after cementation of the occlusal splint and muscle surgery in Group ADR indicates that the mechanisms responsible are in general similar to those in Group A, with the important difference that the effect of stretching of the masseter muscle was largely alleviated. The resulting vector of the elevator muscles of the mandible after stretching in Group A was mainly anteriosuperior. However, because the masseter muscle was stretched only slightly and because it was temporarily incapacitated in Group ADR by the surgery, the chronic forces acting on the mandible in Group ADR differed from those in Group $A$ both in magnitude and direction. The temporalis and medial pterygoid muscles became the principal elevators that were both intact and stretched in Group ADR, perhaps resulting in a more superiorly-directed force acting on the mandible and maxilla. This may have brought about the more superiorly-directed displacement of the maxillary complex and decrease in dental intrusion in Group ADR.

There are a number of mechanisms by which the craniofacial complex in general and its muscles in particular can adapt after stretching of the muscles of mastication [see Carlson et al. (1982) and Carlson and Poznanski (1982) for reviews]. For example, tension created by stretched muscles might actually cause their skeletal attachments to become displaced so that they come closer together, thus allowing the muscle to shorten. Such displacement of osteotomized segments after alteration of muscle origin and insertion is characterized as skeletal relapse. Muscle stretch may also result in longitudinal growth of the fibres through the addition of sarcomeres (Tabary et al., 1972), increased amounts of connective tissue within the muscles and migration of muscles (Hoyte and Enlow, 1966; Carlson et al., 1982). Maxwell et al. (1981) found some evidence to indicate that stretching of the mandibular elevator muscles results in an increase in sarcomere number per fibre; however these data were ambiguous because of differences in body size and small sample sizes for each experimental interval. As the animals in the present study were not killed at its completion, it was not possible to examine whole muscle fibres to demonstrate whether sarcomere number per fibre increased in the experimental animals. The lack of any significant superior displacement of the muscle markers relative to the mandibular bone markers (Fig. 6B) demonstrated that there was no sig- 
nificant interstitial fibre growth distal to the markers within the masseter. However, it is likely that the sum total of the sarcomeres added to fibres in series proximal to the markers was sufficient to increase the length of the masseter muscle. We suggest, therefore, that the tension produced by the stretched muscles and connective tissues resulted in sutural growth leading to displacement of the maxillary complex that continued until a new homeostasis between muscle length, muscle function and skeletal form was established, i.e. until the stretch of the muscles and soft tissues was largely alleviated. As the analysis of masseter muscle position effectively eliminates the possibility of muscle migration in this adaptive response, we propose three mechanisms that most reasonably explain the muscle adaptation: (1) shortening of the stretched masseter due to distraction of the maxillary complex and rotation of the mandible, (2) longitudinal, interstitial muscle fibre growth via addition of sarcomeres and (3) connective tissue growth. The latter two resulted in a permanently lengthened muscle.

Acknowledgements-This research was supported in part by grants DE 05232 and DE 04227 and contract DE 052478 from the National Institutes of Health.

\section{REFERENCES}

Bell W. H. (1977) Correction of the sort-face syndromevertical maxillary deficiency: A preliminary report. $J$. oral Surg. 35, 110-120.

Bell W. H. and Scheideman G. B. (1981) Correction of vertical maxillary deficiency: stability and soft tissue changes. J. oral Surg. 39, 666-670.

Botterman B. R., Binder M. D. and Stuart D. G. (1978) Functional anatomy of the association between motor units and muscle receptors. Am. Zool. 18, 135-152.

Brill N. and Tryde G. (1974) Physiology of mandibular positions. In: Frontiers of Oral Physiology (Edited by Kawamura Y.) pp. 199-237. Karger, Basel.

Carlson D. S., Ellis III E., Schneiderman E. D. and Ungerleider J. C. (1982) Expcrimental models of surgical intervention in the growing face: Cephalometric analysis of facial growth and relapse. In: The Effect of Surgical Intervention on Craniofacial Growth (Edited by McNamara Jr J. A., Carlson D. S. and Ribbens K. A.). Monograph No. 12, Craniofacial Growth Series, Center for Human Growth and Development, The University of Michigan, Ann Arbor, pp. 11-72, 1982.

Carlson D. S. and Poznanski A. (1982) Experimental models of surgical intervention in the growing face: Histochemical analysis of neuromuscular adaptation to altered muscle length. In: Effect of Surgical Intervention on Craniofacial Growth (Edited by McNamara J. A. Jr, Carlson D. S. and Ribbens K. A.) Monograph No. 12, Craniofacial Growth Series, Center for Human Growth and Development. The University of Michigan, Ann Arbor, pp. 73-98.

Carlson F. D. and Wilkie D. R. (1974) Muscle Physiology. Prentice-Hall, Englewood Cliffs, N.J.

Cooker H. S., Larson C. R. and Luschei E. J. (1980). Evidence that the human jaw stretch reflex increases the resistance of the mandible to small displacements. $J$. Physiol, Lond. 308, 61-78.
Dechow P. C. and Carison D. S. (1982a) Development of masticatory muscle force in macaques. $J$. dent. Res. 61. 211

Dechow P. C. and Carlson D. S. (1982b) Bite force and gape in rhesus macaques. Am. J. phys. Anthrop. 57, 179.

Epker B. N., Wolford L. M. and Fish L. C. (1978) Mandibular deficiency syndrome. Surgical considerations for mandibular advancement. Oral Surg. 45, 349-363.

Gantt D. (1979) Patterns of dental wear and the role of the canine in Cerocopithecinae. Am. J. phys. Anthrop. 51. 353-360.

Houk J. C. (1979) Regulation of stiffness by skeletomotor reflexes. A. Rev. Physiol. 41, 99-114.

Hoyte D. A. N. (1966) Experimental investigations of skull morphology and growth. In: International Review of General and Experimental Zoology (Edited by Felts W. J. L. and Harrison R. J.) pp. 345-400. Academic Press, New York.

Hoyte D. A. N. and Enlow D. H. (1966) Wolf's law and the problem of muscle attachment on resorptive surfaces of bone. Am. J. phys. Anthrop. 24. 205-214.

Hurme V. O. and Van Wagenen G. (1961) Basic data on the emergence of permenent teeth in the rhesus monkey. Proc. Am. Phil. Soc. 105, 40.

Ive J., McNeill R. W. and West R. A. (1977) Mandibular advancement: skeletal and dental changes during fixation. J. oral Surg. 35, 881.

Maxwell L. C., Carlson D. S., McNamara Jr J. A. and Faulkner J. A. (1981) Adaptation of the masseter and temporalis muscles following alteration in length, with or without surgical detachment. Anat. Rec, 200, 127-137.

McNamara J. A. Jr. (1974) Histologic and cephalometric responses to increased vertical dimension in the mature face. J. dent. Res. 53, 147.

McNamara J. A. Jr. (1975) The role of muscle and bone interaction in craniofacial growth. In: Control Mechanisms in Craniofacial Growth (Edited by McNamara J. A. Jr) Monogr. No. 3, Craniofacial Growth Series, Center for Human Growth and Development, The University of Michigan, Ann Arbor.

McNamara J. A. Jr. (1977) An experimental study of increased vertical dimension in the growing face. Am. $J$. Orthodont. 71, 382-395.

McNamara J. A., Carison D. S. Yellich G. M. and Hendrickson R. P. (1978) Musculoskeletal adaptation following orthognathic surgery. In: Muscle Adaptation in the Craniofacial Region (Edited by Carlson D. S. and McNamara J. A.) Monograph No. 8, Craniofacial Growth Series, Center for Human Growth and Development, University of Michigan, Ann Arbor, pp. 91-131.

Moore W. J. and Lavelle C. L. B. (1974) Growth of the Facial Skeleton in the Hominoidea. Academic Press, New York.

Poulton D. R. and Ware W. H. (1971). Surgical-orthodontic treatment of severe mandibular retrusion. $A m$. J. Orthod. 59, 244-265.

Schneiderman E. D. and Carlson D. S. (1981) Growth and remodeling of the mandible following alteration of function in adult rhesus monkeys. Am. J. phys. Anthrop. 54, 275.

Steinhauser E. W. (1973) Advancement of the mandible by sagittal ramus split and suprahyoid myotomy. J. oral Surg. 31, 516-521.

Tabary J. C., Tabary C., Tardieu C., Tardieu G. and Goldspink G. (1972) Physiological and structural changes in the cat's soleus muscle due to immobilization at different lengths by plaster casts. J. Physiol., Lond. 224, 231-244.

Ware W. H. and Taylor R. C. (1968) Condylar repositioning following osteotomies for correction of mandibular prognathism. Am. J. Orthod. 54, 50-59.

Washburn S. L. (1947) The relation of the temporal muscle to the form of the skull. Anat. Rec. 99, 239-248. 
Willmar K. (1974) On Le Fort 1 Osteotomy. Scand. J. Plas. Yemm R. and Berry D. C. (1969) Passive control in manRecon. Surg. Suppl. 12.

dibular rest position. $J$. prosth. Dent. 22, 30-36.

Yellich G. M., McNamara J. A. Jr and Ungerleider J. C. Yemm R. and Nordstrom S. H. (1974) Forces developed by (1981) Muscular and skeletal adaptations following lengthening, detachment and reattachment of the mastissue elasticity as a determinant of mandibular resting seter muscle. J. oral Surg. 39, 656-665. posture in the rat. Archs oral Biol. 19, 347-351. 
Plate 1.

Fig. 1. Lateral cephalograms of an adult female rhesus monkey immediately prior to (A) and after (B) cementation of the bite-opening appliance. $\mathrm{b}$, Bone implant; $\mathrm{m}$, muscle implant; appl, the appliance. 


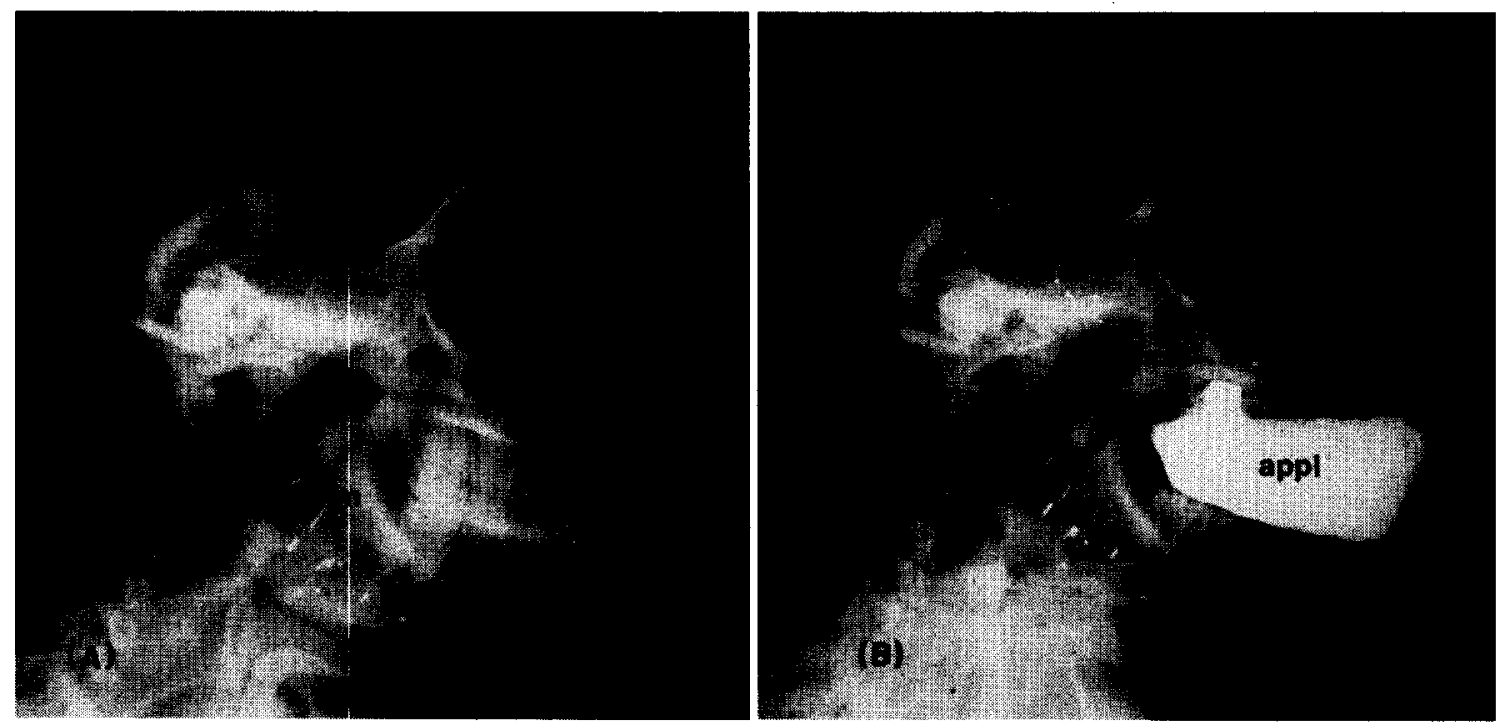

Plate 1. 